\title{
Diversité urbaine
}

Introduction

Introduction

\section{Gabrielle Désilets et Sandrine Jean}

Volume 19, 2019

La mobilité des migrants middling

URI : https://id.erudit.org/iderudit/1065117ar

DOI : https://doi.org/10.7202/1065117ar

Aller au sommaire du numéro

\section{Éditeur(s)}

Groupe de recherche diversité urbaine

ISSN

1913-0708 (numérique)

Découvrir la revue

Citer ce document

Désilets, G. \& Jean, S. (2019). Introduction. Diversité urbaine, 19, 5-9.

https://doi.org/10.7202/1065117ar d'utilisation que vous pouvez consulter en ligne.

https://apropos.erudit.org/fr/usagers/politique-dutilisation/ 


\section{Introduction}

\section{GABRIELLE DÉSILETS}

Concordia University, gabrielle.desilets@concordia.ca

\section{SANDRINE JEAN}

Memorial University of Newfoundland, sjean@mun.ca

$\mathrm{L}$ ES DERNIÈRES DÉCENNIES ont été marquées par l'augmentation de l'octroi de visas temporaires, au détriment de ceux accordant le statut permanent, ainsi que par la multiplication des types de visas temporaires dans les pays traditionnels d'immigration comme le Canada. Par exemple, à partir de 2006, le nombre de résidents temporaires admis au pays dépassait le nombre de résidents permanents sur le territoire (Citoyenneté et Immigration Canada 2015). Le recours aux travailleurs temporaires et aux migrants qualifiés marque un tournant dans la politique migratoire canadienne (Alboim et Cohl 2012 ; Foster 2012 ; Piché 2012). Néanmoins, peu d'études se sont penchées sur l'expérience de migration des travailleurs temporaires qualifiés (Dauvergne et Marsden 2014; Kuvik 2012). La catégorie de visa et le type de qualification avec lesquels une personne arrive dans une nouvelle société sont déterminants dans l'expérience vécue de la migration. Cependant, le statut légal et les qualifications à eux seuls ne suffisent en aucun cas pour expliquer la diversité des trajectoires migratoires des personnes aujourd'hui en situation de mobilité. Les articles de ce numéro illustrent la complexité et la circularité des flux migratoires contemporains ainsi que la multiplicité des parcours des migrants appartenant à la classe moyenne. Les données de terrain rassemblées montrent qu'en observant l'intersection entre ces catégorisations, on constate toute une gamme de statuts qui ne sont pas fixes, mais hautement mobiles et contrastés dans le temps et dans l'espace.

Dans ce numéro thématique, nous nous intéressons à la mobilité volontaire et souvent temporaire des migrants middling, un terme d'abord utilisé par Conradson et Latham (2005) pour nommer la mobilité des 
personnes qui appartiennent à la «classe moyenne» transnationale, soit dans le pays de départ, soit dans celui d'arrivée, ou encore dans les deux (Smith 2005). Cette «nouvelle classe» de migrants aux origines sociales, ethniques et nationales diverses (Meier 2014) se distingue de celle des migrants «forcés». En effet, les middlings choisissent volontairement et personnellement de migrer, souvent dans le but de travailler à l'étranger. Selon Lasch (1995), les professionnels transnationaux forment une «nouvelle classe», étant donné que leurs activités de subsistance dépendent non pas du principe de propriété, mais plutôt de leur capacité à manipuler l'information et l'expertise professionnelle dans l'économie du savoir et de la connaissance. Dans ce que Plöger et Becker (2015) qualifient de skills turn, la migration des middlings s'inscrit aussi dans les stratégies déployées par les États, les régions et les villes des pays industrialisés pour attirer et retenir les travailleurs qualifiés en contexte de vieillissement des populations et de pénurie de main-d'œuvre. Pourtant, au-delà de ces discours politisés, les parcours migratoires des middlings ne sont pas exempts d'obstacles (Rutten et Verstappen 2014). Nous en savons encore peu sur les motifs de leur migration, sur leurs expériences à l'arrivée dans le pays d'accueil ainsi que sur leur quotidien dans les villes où ils s'installent. Les quatre articles de ce numéro thématique traitent de cette question.

L'article de Gabrielle Désilets présente des données tirées d'une recherche ethnographique dans les rues, les commerces et les entreprises de l'écosystème créatif du secteur Saint-Viateur Est du Mile-End, à Montréal. L'auteure présente la catégorie des migrants middling par rapport à celles de l'élite mobile et des travailleurs non qualifiés pour offrir une lecture critique des politiques migratoires qui misent sur les stratégies d'attraction et de rétention des travailleurs qualifiés. En exposant les contradictions inhérentes entre les motivations personnelles et les incitatifs structurels propres au marché du travail mondialisé, l'auteure mobilise le concept de temporalité ainsi que l'éthos de la «classe moyenne globale» pour soutenir que ces variables maintiennent les travailleurs rencontrés dans un entre-deux entre mobilités et ancrages. Larticle contribue au champ de l'urbanisme transnational en exposant la spécificité historique et locale du quartier Mile-End, à Montréal, et en démontrant de quelle manière ces travailleurs temporaires qualifiés transforment l'espace urbain pour forger leur appartenance à un mode de vie globalisé.

Dans son article sur l'entrepreneuriat de petite taille en contexte de mobilité «Nord-Sud», Émilie Angrignon-Girouard propose une réflexion sur l'éthos entrepreneurial de jeunes adultes européens qualifiés dans la ville de Mexico. S'appuyant sur une étude ethnographique menée auprès de ces jeunes migrants, elle démontre de quelle manière leurs 
choix migratoires et professionnels sont influencés par l'importance accrue accordée à leur quête d'individualité. En présentant trois cas, l'auteure met en exergue de nouvelles manières d'appréhender le projet entrepreneurial dans lequel l'autonomisation et la responsabilisation de l'entrepreneur sont centrales. Contrairement aux analyses classiques de l'entrepreneuriat en contexte migratoire qui mise sur les liens familiaux, ethniques et transnationaux des personnes, ces résultats suggèrent plutôt que l'impulsion principale de l'activité économique de ces jeunes de classe moyenne est la réalisation du soi individuel portée par un éthos entrepreneurial ancré dans les sociétés post-industrielles, et qui valorise l'autonomie, la créativité et l'innovation.

Larticle de Danièle Bélanger, Myriam Ouellet et Charles Fleury traite des parcours migratoires et des expériences de 26 travailleurs temporaires qualifiés au Québec. Les auteurs présentent une analyse critique de la notion de précarité et de «temporairité » des statuts d'immigration dans des pays d'immigration, tels que le Canada, qui ont largement recours aux programmes de travail temporaire pour combler les besoins de maind'œuvre. En situant leur analyse dans le contexte d'un changement d'idéologie régissant la politique migratoire canadienne en faveur des résidents temporaires plutôt que des résidents permanents, les auteurs mettent en évidence le fossé entre la rhétorique gouvernementale vantant les mérites de la migration de travail temporaire et l'absence de services et d'interlocuteurs étatiques pour ces «temporaires», contribuant ainsi au statut ambigu et d'entre-deux vécu par ces migrants. En appliquant le gradient de précarité proposé par Goldring et al. (2009), ils font le constat d'une double précarité, à la fois en tant que résidents et en tant que travailleurs temporaires. Si ces migrants sont souvent perçus comme une catégorie de travailleurs volontaires et privilégiés en comparaison avec d'autres catégories de migrants, les résultats présentés dans cet article révèlent la vulnérabilité insoupçonnée de ce groupe de travailleurs.

Larticle de Monica Schlobach aborde le processus de prise de décision entourant l'émigration d'ingénieurs brésiliens au Québec. En s'appuyant sur une collecte de récits de vie et d'activités d'observation participante, l'auteure détaille le parcours migratoire d'un couple d'ingénieurs brésiliens. Schlobach avance l'hypothèse que le projet migratoire des migrants qualifiés repose sur l'acquisition et l'utilisation de multiples capitaux. De plus, la mobilisation des réseaux sociaux virtuels joue un rôle de premier plan dans leur processus d'émigration. S'inspirant des théories de Bourdieu (1986), l'auteure présente le «capital cognitif», manifesté dans la mobilisation de l'habitus professionnel, le «capital social», inscrit dans les réseaux sociaux, le «capital culturel» et finalement le «capital économique » comme étant les ressources migratoires tour à tour mobili- 
sées par ces migrants qualifiés. L'analyse révèle que la migration se décide d'abord dans la société de départ, se construit à partir des réseaux sociaux des migrants dans la société d'origine et se prépare par la mobilisation et la création de nouvelles ressources, dont les réseaux sociaux virtuels établis avec les immigrants dans la société d'arrivée.

Le numéro compte également deux articles hors thème. L'article de Marie-Odile Magnan, Annie Pilote, Tya Collins et Pierre Canisius Kamanzi porte sur les inégalités scolaires perçues par des élèves dont les parents sont nés dans les Caraïbes ou en Amérique latine. Il vise à documenter les effets de l'environnement et de l'action scolaire sur leur parcours scolaire. S'appuyant sur une analyse inductive et rétrospective des récits de vie de 24 jeunes fréquentant un établissement postsecondaire à Montréal, les auteurs soulignent le rôle du système éducatif et de l'école dans la construction des inégalités de persévérance et dans la persistance des discriminations systémiques à l'égard de ces jeunes issus de l'immigration. Larticle propose des pistes de réflexion pour favoriser l'équité en milieu scolaire face à l'indifférence institutionnelle et à l'exclusion par le groupe majoritaire.

Finalement, l'article de Luisa Veronis et Suzanne Huot utilise le concept d'imaginaire géographique pour examiner le rôle des représentations sociales et spatiales des communautés francophones en situation minoritaire au Canada pour les immigrants et les réfugiés d'expression française. Dans une perspective postcoloniale, elles y abordent les processus d'inclusion et d'exclusion entre francophones immigrants et non immigrants, et les relations de pouvoir entre groupes dominants et dominés. Leur article s'appuie sur des entrevues et des groupes de discussions avec des immigrants francophones dans deux villes ontariennes: London et Ottawa. L'analyse met en lumière les dynamiques sociospatiales de la francophonie minoritaire canadienne telles qu'elles sont perçues par les immigrants francophones et leur rôle dans la négociation de leur appartenance.

Au nom de l'équipe de Diversité urbaine et des auteurs de ce numéro, nous vous souhaitons une bonne lecture!

\section{Bibliographie}

Alboim, N. et K. Cohl, 2012. "Shaping the Future: Canada's Rapidly Changing Immigration Policies», Maytree Fondation. https://maytree.com/wp-content/ uploads/shaping-the-future.pdf [consulté le 15 avril 2018].

Bourdieu, P., 2011 [1986]. «The Forms of Capital», in I. Szeman et T. Kaposy (dir.), Cultural Theory: An Anthology. Malden, Wiley-Blackwell, p. 81-93.

Citoyenneté et Immigration Canada, 2015. Faits et chiffres 2015: aperçu de l'immigration - résidents temporaires. Ottawa, Citoyenneté et Immigration Canada. http:// 
www.cic.gc.ca/francais/pdf/2014-Faits-chiffres-Temporaire.pdf [consulté le 9 septembre 2016].

Conradson, D. et A. Latham, 2005. «Transnational Urbanism: Attending to Everyday Practices and Mobilities », Journal of Ethnic and Migration Studies, vol. 31, n ${ }^{\circ}$, p. 227-233.

Dauvergne, C. et S. Marsden, 2014. «The Ideology of Temporary Labour Migration in the Post-Global Era», Citizenship Studies, vol. 18, n 2, p. 224-242.

Foster, J., 2012. «Making Temporary Permanent: The Silent Transformation of the Temporary Foreign Worker Program», Just Labour, vol. 19, p. 22-46.

Goldring, L., C. Berinstein et J. K. Bernhard, 2009. «Institutionalizing Precarious Migratory Status in Canada», Citizenship Studies, vol. 13, n 3, p. 239-265.

Kuvik, A., 2012. «Skilled Migration in Europe and Beyond: Recent Developments and Theoretical Considerations », in M. Martiniello et J. Rath (dir.), An Introduction to International Migration Studies: European Perspectives. Amsterdam, Amsterdam University Press, p. 211-235.

Lasch, C., 1995. The Revolt of the Elites and the Betrayal of Democracy. New York, Norton.

Meier, L., 2014. Migrant Professionals and the City: Local Encounters, Identities and Inequalities. New York, Routeledge.

Piché, V., 2012. «In and Out the Back Door: Canada's Temporary Worker Programs in a Global Perspective», in M. Geiger et A. Pécoud (dir.), The New Politics of International Mobility. Migration Management and its Discontents. Onasbrück, Université d'Onasbrück, p. 113-132.

Plöger, J. et A. Becker, 2015. «Social Networks and Local Incorporation - Grounding High-skilled Migrants in Two German Cities», Journal of Ethnic E Migration Studies, vol. 41, n 10, p. 1517-1535.

Rutten, M. et S. Verstappen, 2014. «Middling Migration: Contradictory Mobility Experiences of Indian Youth in London », Journal of Ethnic and Migration Studies, vol. 40, n 8, p. 1217-1235.

Scott, S., 2006. «The Social Morphology of Skilled Migration: The Case of the British Middle Class in Paris», Journal of Ethnic and Migration Studies, vol. 32, n ${ }^{\circ}$, p. 1105-1129.

Smith, M. P., 2005. «Transnational Urbanism Revisited», Journal of Ethnic and Migration Studies, vol. 31, nº 2, p. 235-244. 